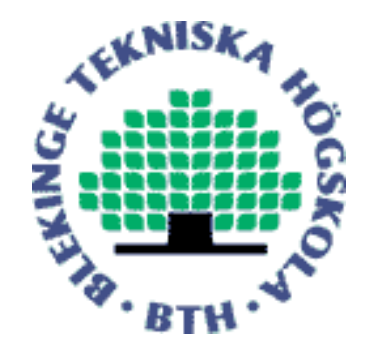

Copyright @ 2013 IEEE.

Citation for the published paper:

Title:

Adaptive Fingerprint Image Enhancement With Emphasis on Preprocessing of Data

Author:

Josef Strom Bartunek, Mikael Nilsson, Benny Sällberg, Ingvar Claesson

Journal:

IEEE Transactions on Image Processing

Year:

2013

Vol:

22

Issue:

2

Pagination:

644-656

URL/DOI to the paper:

10.1109/TIP.2012.2220373

This material is posted here with permission of the IEEE. Such permission of the IEEE does not in any way imply IEEE endorsement of any of BTH's products or services Internal or personal use of this material is permitted. However, permission to reprint/republish this material for advertising or promotional purposes or for creating new collective works for resale or redistribution must be obtained from the IEEE by sending a blank email message to pubs-permissions@iee.org.

By choosing to view this document, you agree to all provisions of the copyright laws protecting it. 


\title{
Adaptive Fingerprint Image Enhancement with Emphasis on Pre-processing of Data
}

\author{
Josef Ström Bartůněk ${ }^{1}$, Student Member, IEEE, Mikael Nilsson ${ }^{2}$, Member, IEEE, \\ Benny Sällberg ${ }^{1}$, Member, IEEE, and Ingvar Claesson ${ }^{1}$, Member, IEEE,
}

\begin{abstract}
This article proposes several improvements to an adaptive fingerprint enhancement method that is based on contextual filtering. The term adaptive implies that parameters of the method are automatically adjusted based on the input fingerprint image. Five processing blocks comprise the adaptive fingerprint enhancement method, where four of these blocks are updated in our proposed system. Hence, the proposed overall system is novel. The four updated processing blocks are; preprocessing, global analysis, local analysis and matched filtering. In the pre-processing and local analysis blocks, a nonlinear dynamic range adjustment method is used. In the global analysis and matched filtering blocks, different forms of order statistical filters are applied. These processing blocks yield an improved and new adaptive fingerprint image processing method. The performance of the updated processing blocks is presented in the evaluation part of this paper. The algorithm is evaluated towards the NIST developed NBIS software for fingerprint recognition on FVC databases.
\end{abstract}

Index Terms-Image processing, successive mean quantization transform, directional filtering, fourier transform, spectral feature estimation.

\section{INTRODUCTION}

Until the 1960's, fingerprint matching was used solely for forensic purposes and human experts performed the fingerprint analysis manually. Research has been conducted the last 50 years to develop automatic fingerprint identification systems (AFIS) (see, e.g., [1] for a survey on AFIS methods). However, fingerprint matching, especially when the fingerprint images have low quality or when the matching is performed crosssensors, is still an open research question. The main problem in automatic fingerprint identification is to acquire matching reliable features from fingerprint images with poor quality.

Contextual filtering is a popular technique for fingerprint enhancement, where topological filter features are aligned with the local orientation and frequency of the ridges in the fingerprint image. The first method utilizing contextual filters to enhance fingerprint images performed both the filter design and the filtering in the spatial domain [2], [3]. The method used a main filter having a horizontally directed pattern designed based on four manually identified parameters for each fingerprint image. Additional directions were created by rotating the

Copyright (C)2012 IEEE. Personal use of this material is permitted. However, permission to use this material for any other purposes must be obtained from the IEEE by sending a request to pubs-permissions@ieee.org.

The Authors ${ }^{1}$ are with Department of Electrical Engineering, The School of Engineering, Blekinge Institute of Technology, SE-371 79 Karlskrona, Sweden, jsb@bth.se, bsa@bth.se, icl@bth.se.

The Authors ${ }^{2}$ are with Centre for Mathematical Sciences, Faculty of Engineering LTH, University of Lund, Sweden, micken@maths. 1 th. se. main horizontal filter while the filter size remained constant. Other fingerprint enhancement methods employ directional Gabor or Butterworth bandpass filters where the filtering is performed in the frequency domain [4], [5]. Second directional derivatives for filter design, and a method for selecting a suitable size of the local area, were presented in [6]. Recently, a method based on curved Gabor filters that locally adapts the filter shape to the curvature and direction of the flow of the fingerprint ridges was introduced in [7]. This new type of Gabor filter design, shows a potential in fingerprint image enhancement in comparison to classical Gabor filter methods. However the computational load is immense which inhibits its use in practical applications.

Another method that stands out from the classical directional filter design approaches was proposed in [8]. Instead of requiring tuned parameters for each fingerprint image, the magnitude spectrum of each local area of the fingerprint image was directly used to filter the same local area in the frequency domain. The rationale behind this method is that the local magnitude spectrum carries properties similar to a matched filter, and by using the magnitude spectrum directly as a filter, dominant components related to ridges are amplified. It is noted that this approach provides a noise gain as well, which makes it less useful in practical situations.

Existing methods typically keep various parameters, such as local area size, constant. The strategy to keep parameters constant may fail in a real application where fingerprint image or sensor characteristics vary, thus yielding varying image quality. In addition, due to the spatially variable nature of fingerprints, it is crucial to have a sufficient amount of data in each local image area so that the local structure of the fingerprint is enclosed. Hence, the local area size should adapt to the data present. Different fingerprint sensor resolutions provide different normalized spatial frequencies of the same fingerprint and this also requires adaptive parameters. Fingerprints captured with the same sensor may also vary depending on, e.g., gender and age of the user (see an example in Fig.1). The negative influence on fingerprint recognition system performance for individuals of different ages was demonstrated in [9] and the matching results of Db3 in FVC2000 [10]. To compensate for varying fingerprint image characteristics and to achieve an optimal system performance, key parameters of most existing methods, e.g., the size of the local area, need to be tuned manually for every fingerprint image. This manual tuning for each image is tedious and costly and automatic systems are therefore desirable.

This article extends an existing adaptive fingerprint en- 


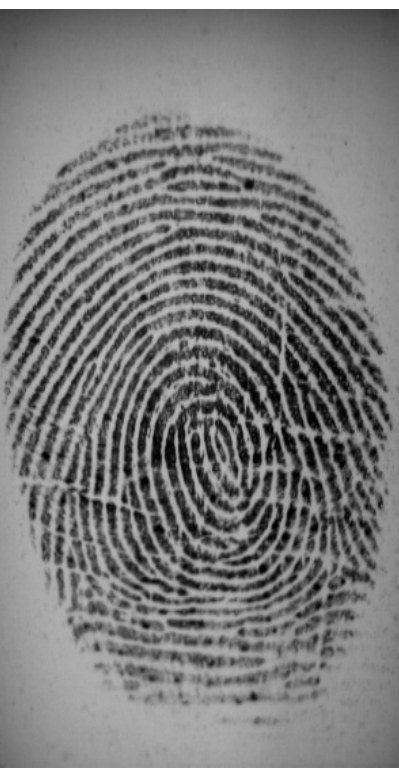

(a)

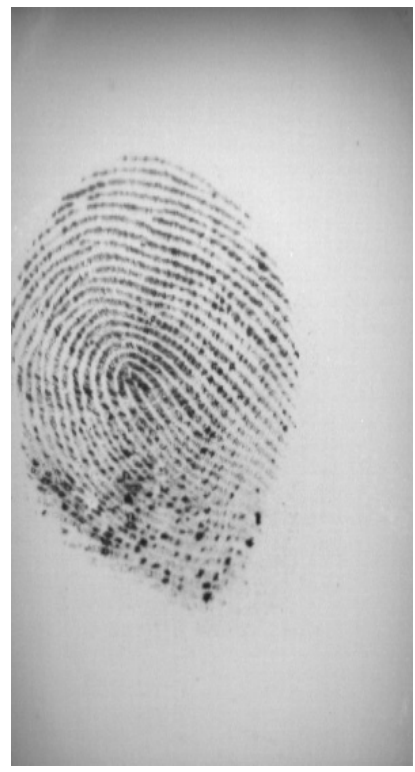

(b)
Fig. 1. Fingerprint sensor images of the little finger of a 30 years old man (a), and the little finger of a 5 years old boy (b), illustrates the varying fingerprint image quality.

hancement system (presented in [11], [12]) by incorporating new processing blocks to construct an improved novel system. The proposed fingerprint enhancement method is based on spatial contextual filtering using matched directional filters. A non-linear dynamic range adjustment method is used as a preprocessing stage in this paper. An outlier suppression using a median filter is proposed in the global spectral analysis to further improve the estimation of the fingerprint's fundamental frequency. The median filter suppresses noise and it has also a grouping effect which aids the frequency estimation. The present method furthermore proposes to use local dynamic range adjustment to improve local spectral features estimation, and where order statistical filters are used to smooth the spectral features yielding a robust algorithm behavior. The evaluation focuses on the complete assessment of the proposed method's performance using the NBIS software for fingerprint recognition developed by NIST [13] on a three FVC databases [14], [15], [16].

The paper is outlined as follows. The proposed method is introduced in section II. An evaluation is conducted in section III. A discussion is provided in section IV, and a summary with conclusions is given in section V.

\section{A. Abbreviations}

Frequently occurring abbreviations in this paper are presented here:

$\begin{array}{ll}\text { AAC } & \text { Area Above Curve } \\ \text { DET } & \text { Detection Error Tradeoff } \\ \text { EER } & \text { Equal Error Rates } \\ \text { FMR } & \text { False Match Rate } \\ \text { FNMR } & \text { False Non Match Rate } \\ \text { FMR100 } & \text { FNMR when FMR is } 1.0 \% \\ \text { FMR1000 } & \text { FNMR when FMR is } 0.1 \% \\ \text { ZeroFMR } & \text { FNMR when FMR is } 0.0 \%\end{array}$

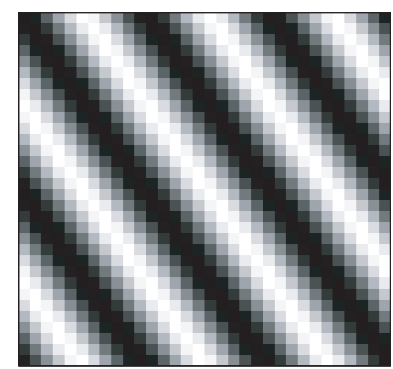

(a)

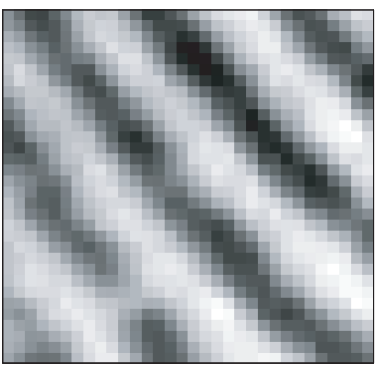

(c)

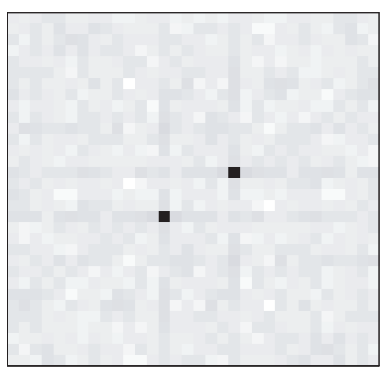

(b)

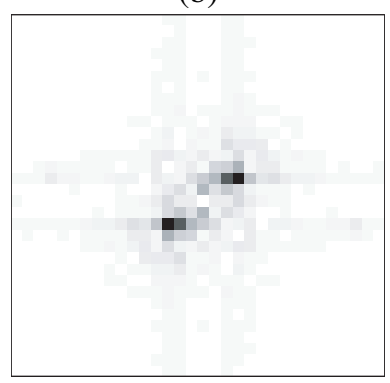

(d)
Fig. 2. A sinusoidal signal (a) and its corresponding magnitude spectrum (b). A local area inside a fingerprint image (c) and its corresponding magnitude spectrum (d). The spatial and spectral similarities between the sinusoidal signal and the fingerprint are used as a fundament in the proposed method.

FVC Fingerprint Verification Contest

NIST National Institute of Standards and Technology

NBIS NIST Biometric Image Software

SMQT Successive Mean Quantization Transform

\section{PROPOSED METHOD}

A spatial sinusoidal signal and its corresponding magnitude spectrum is illustrated together with a local fingerprint image patch and its corresponding magnitude spectrum in Fig. 2. This example is used to state the following observations:

1) Local fingerprint image patches are spatially and spectrally similar to a sinusoidal signal, where the dominant peaks in the magnitude spectrums of the two signals are co-located.

2) The location of the dominant peak in the magnitude spectrum of a local image area carries information about the local orientation and frequency of the fingerprint pattern.

3) The magnitude of the dominant spectral peak acts as an indicator of the quality of the fingerprint in that particular local area [11] (see Fig. 10).

These observations act as a fundament to the method in this paper where the location and magnitude of the dominant local spectral peak are utilized for designing matched directional filters [11], [12]. The contextual filtering and a background segmentation are then performed in the spatial domain based on the extracted local features.

The proposed fingerprint enhancement method is based on an existing method [11], [12]. However, key processing blocks are updated by additional new processing stages so as to yield a novel enhancement system, see Fig. 3. First, an innovative non-linear pre-processing block adjusts the dynamic range of 


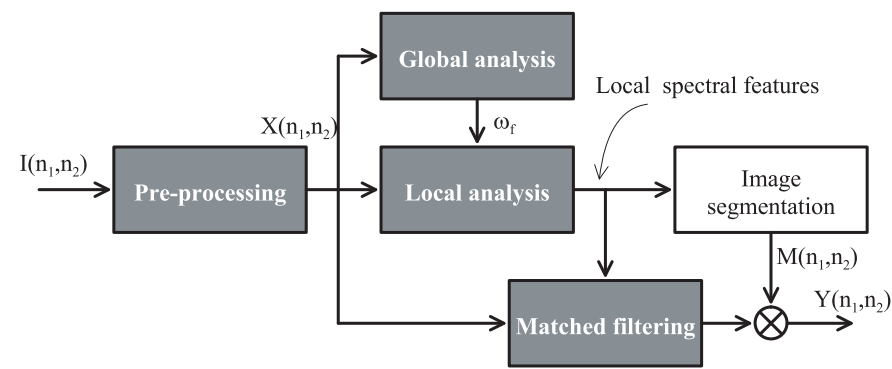

Fig. 3. Processing blocks of the proposed method, where blocks in gray notate updated processing blocks.

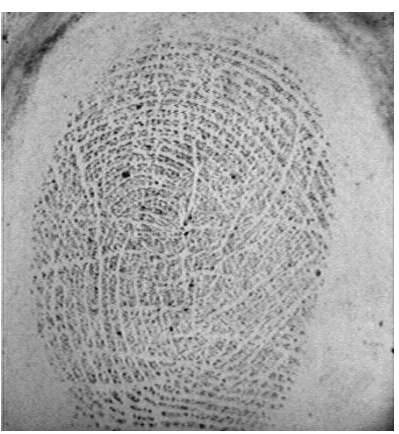

(a)

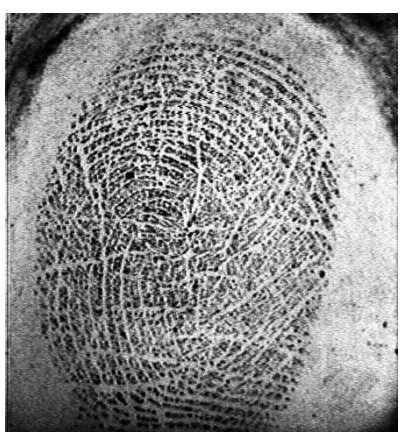

(b)
Fig. 4. Fingerprint image (a), and corresponding SMQT enhanced image (b). Both images have eight bit dynamic range.

the image (see section II-A). Second, a novel update to the previously derived global fingerprint analysis is conducted to aid the fundamental spatial frequency estimation of the fingerprint image, and where a data-outlier suppression further improves the frequency estimation performance for noisy images (see section II-B).

Third, based on the estimated fundamental frequency from the global analysis, a local adaptive analysis (see section II-C) adjusts the fundamental frequency to match the local image area. The local analysis proposes the use of a local dynamic range adjustment method to further improve spectral features estimation. Fourth, the matched filtering is based on the spectral features estimated in the local analysis, where an additional order-statistical filtering of the spectral features is introduced to increase the method's resilience towards noise (see section II-D). Finally, an image segmentation separates fingerprint data from the background (see section II-E). This, taken all together, comprises the proposed new fingerprint enhancement system that automatically tunes its parameters according to each individual fingerprint image.

\section{A. Pre-processing}

Let $I\left(n_{1}, n_{2}\right)$ represent a fingerprint image of size $N_{1} \times N_{2}$, where $n_{1} \in\left[0, N_{1}-1\right]$ and $n_{2} \in\left[0, N_{2}-1\right]$ denote horizontal and vertical coordinates, respectively. Without loss of generality, each element of $I\left(n_{1}, n_{2}\right)$ is assumed to be quantized in 256 gray-scale levels, i.e., the dynamic range of the image is eight bits. However, the fingerprint image may not use the full dynamic range in a practical situation and this may degrade system performance. The Successive

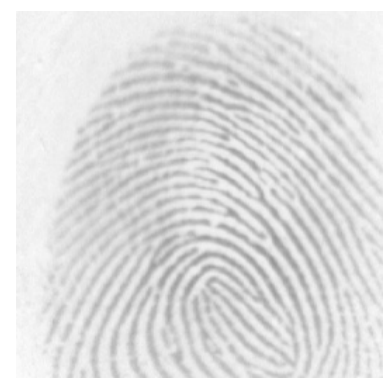

(a)

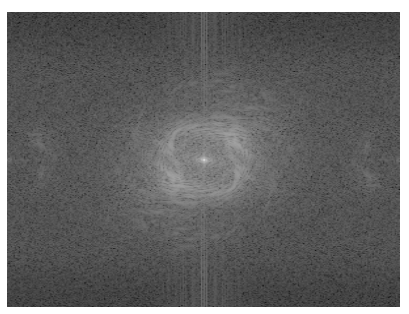

(b)
Fig. 5. Fingerprint image (a) and corresponding magnitude spectrum (b). The circular structure around the origin in the fingerprint magnitude spectrum stems from the characteristics of the periodic fingerprint pattern.

Mean Quantization Transform (SMQT) [17], [18] is used as a dynamic range adjustment in this paper (see an example in Fig. 4). The SMQT can be viewed as a binary tree build of a simple Mean Quantization Units (MQU) where each level performs an automated break down of the information. Hence, with increasing number of levels the more detailed underlying information in the image is revealed. This is equivalent to a nonlinear histogram stretch while still preserving basic histogram shape. This nonlinear property of SMQT yields a balanced image enhancement. Thus, the SMQT adjusts the dynamic range adaptively and nonlinearly and it is configured by only one design parameter $B$. The parameter $B$ corresponds to the number of levels in the binary tree and is equal to the number of bits used to represent the SMQT processed image. The nonlinear SMQT-operation is denoted as $\operatorname{SMQT}_{B}\{\}$. The parameter is set to $B=8$ in the pre-processing stage of this paper, which means that the dynamic range adjustment provided by the SMQT-operation does not alter the bit-depth of the enhanced fingerprint image. The pre-processed eight-bit SMQT image is denoted as $X\left(n_{1}, n_{2}\right)=\mathrm{SMQT}_{8}\left\{I\left(n_{1}, n_{2}\right)\right\}$, where the notation $X$ means that this enhanced image acts as input to further processing.

Large regional contrast variation is quite typical for low quality fingerprint images which require a high dynamic range usage in order to not embed fingerprint ridges in the background. Hence, the SMQT-enhancement is performed using eight bits so as to avoid the risk of obstructing important data in heavily noisy fingerprint images. In addition, the eight-bit SMQT used in the pre-processing requires only a fractional amount of processing as opposed to other parts of the proposed method (see Table II). Optimizing the processing load on this part of the algorithm yields therefore only an insignificant reduction of processing power but increases the risk of reduced performance.

The employment of the SMQT algorithm for fingerprint enhancement with $B=8$ has been previously proposed in [19]. While the idea is novel and is equivalent to the preprocessing step in this paper, it lacks an evaluation on fingerprint image databases with relevant metrics such as EER. Hence, it does not provide any major contribution in relation to [18] which proposes SMQT for arbitrary grayscale image enhancement. 


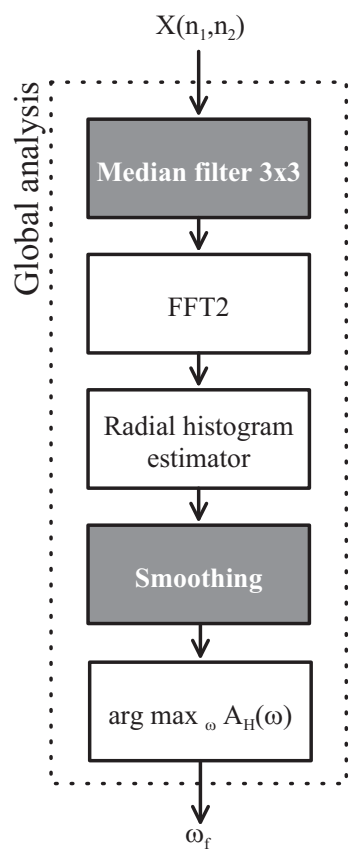

Fig. 6. Processing blocks of the global analysis, where blocks in gray notate novel processing blocks.

\section{B. Global Analysis}

The magnitude spectrum of a fingerprint image typically contains a circular structure around the origin, see the example in Fig. 5. The circular structure stems from the fact that a fingerprint has nearly the same spatial frequency throughout the image but varying local orientation. The circular structure in the magnitude spectrum has been used for estimating fingerprint quality in [20], [21]. In a recent study, the circular spectral structure was exploited to detect the presence of a fingerprint pattern in the image [22]. This paper employs that the radially dominant component in the circular structure corresponds to the fundamental frequency of the fingerprint image. This fundamental frequency is inversely proportional to a fundamental window size which is used as a base window size in our method.

The fundamental fingerprint frequency is estimated in the global analysis according to the following steps (see a block schema in Fig. 6):

1) A new processing stage suppresses data outliers by a median filter.

2) A radial frequency histogram is computed from the magnitude spectrum of the median filtered image.

3) The fundamental frequency of the fingerprint is assumed located at the point where the radial frequency histogram attains its maximal value. The radial frequency histogram is herein proposed to be smoothed in order to reduce the impact of spurious noise.

1) Step 1 - Data-outlier suppression: This paper proposes to apply a $3 \times 3$ median filter to the SMQT enhanced image in order to suppress data outliers. The median filtered fingerprint image is denoted as $Z\left(n_{1}, n_{2}\right)=\operatorname{Median}_{3 \times 3}\left\{X\left(n_{1}, n_{2}\right)\right\}$.

2) Step 2 - Radial frequency histogram: Let $F\left(\omega_{1}, \omega_{2}\right)=$ $\mathfrak{F}\left\{Z\left(n_{1}, n_{2}\right)\right\}$ denote the two-dimensional Fourier transform

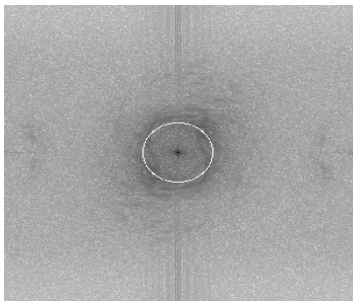

(a)

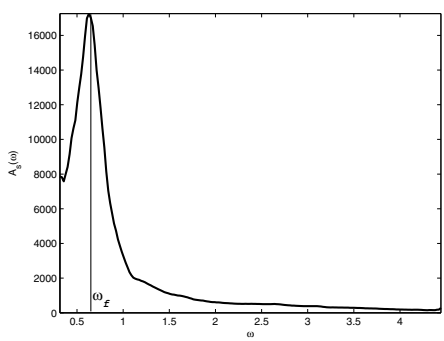

(b)
Fig. 7. Fingerprint magnitude spectrum with an overlayed circle whose radius corresponds to the estimated fundamental frequency $\omega_{f}$ (a) and the corresponding radial frequency histogram $A_{s}(\omega)$ whose peak value is located at the fundamental frequency (b).

(see, e.g., [23]) of the pre-processed and median filtered input image $Z\left(n_{1}, n_{2}\right)$, where, $\omega_{1} \in[-\pi, \pi)$ and $\omega_{2} \in[-\pi, \pi)$ denote normalized frequency. The spectral image is represented in polar form for clarity in the presentation, i.e., $F\left(\omega_{1}, \omega_{2}\right) \equiv F(\omega, \theta)$, related through the following change of variables $\omega_{1}=\omega \cdot \cos \theta$ and $\omega_{2}=\omega \cdot \sin \theta$, where $\omega$ is the normalized radial frequency and $\theta$ denotes the polar angle.

A radial frequency histogram $A(\omega)$ is obtained by integrating the magnitude spectrum $|F(\omega, \theta)|$ along the polar angle $\theta$, according to

$$
\begin{aligned}
A(\omega) & =\frac{1}{2 \pi} \int_{0}^{2 \pi}|F(\omega, \theta)| d \theta \\
& =\frac{1}{\pi} \int_{0}^{\pi}|F(\omega, \theta)| d \theta,
\end{aligned}
$$

where, due to the complex conjugate symmetry of $F(\omega, \theta)$, it is sufficient to integrate only over one half-plane in Eq. 1.

3) Step 3 - Fundamental frequency estimation: The radial frequency histogram may contain impulsive noise due to noisy input signals. This paper therefore proposes employing a smoothing filter (smoothing along the $\omega$-variable in $A(\omega)$ ) to suppress the impulsive noise, where the smoothed radial frequency histogram is denoted as $A_{S}(\omega)$. The radial frequency at the point where the radial frequency histogram attains its largest value corresponds to the fundamental frequency $\omega_{f}$ of the fingerprint image, i.e.,

$$
\omega_{f}=\arg \max _{\omega \in\left[\omega_{\min }, \pi\right]} A_{S}(\omega) .
$$

The lower boundary $\omega_{\min }$ is introduced in order to avoid erroneous peak values related to low frequency noise. Empirical analysis shows that there are at least 10 full periods of the fingerprint pattern in an image. Hence, the lower search boundary is computed as

$$
\omega_{\min }=\frac{2 \cdot \pi \cdot 10}{\max \left(N_{1}, N_{2}\right)} .
$$

For practical reasons, the radial frequency is made discrete in the implementation, and a five point FIR filter with the Ztransform $H(z)=\frac{1}{5}\left(1+z^{-1}+z^{-2}+z^{-3}+z^{-4}\right)$ is used to smooth the radial frequency histogram. One example of a fingerprint magnitude spectrum together with a corresponding radial frequency histogram is illustrated in Fig. 7. 


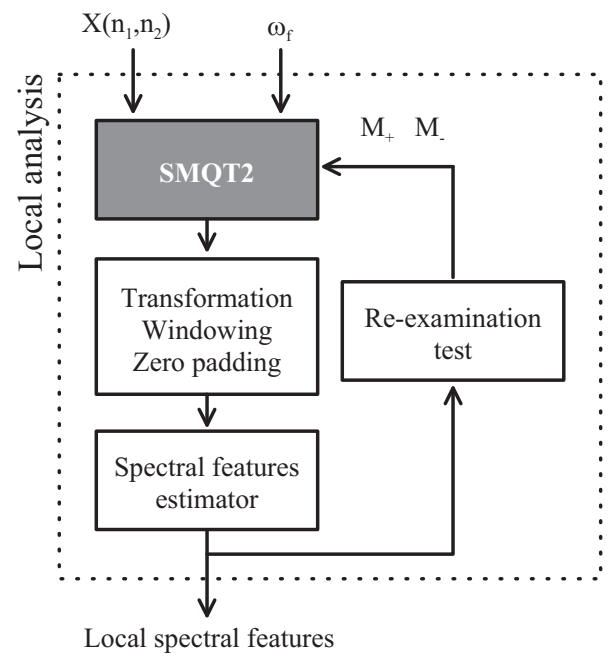

Fig. 8. Processing blocks of the local adaptive analysis, where blocks in gray notate novel processing blocks.

The fundamental frequency $\omega_{f}$, computed in Eq. 2, is inversely proportional to a fundamental area size $L_{f}$, according to

$$
L_{f}=\frac{2 \pi}{\omega_{f}} .
$$

The major advantage of the method proposed in this paper is that it is adaptive towards sensor and fingerprint variability. The adaptive behavior is due to that the estimated fundamental area size acts as a base window size in all stages of the method. Hence, no parameter tuning is required to use the proposed method for different sensors or applications.

\section{Local Adaptive Analysis}

The purpose of the local analysis is to adaptively estimate local spectral features corresponding to fingerprint ridge frequency and orientation. Most parts of a fingerprint image containing ridges and valleys have, on a local scale, similarities to a sinusoidal signal in noise. Hence, they have a magnitude spectrum with two distinct spectral peaks located at the signal's dominant spatial frequency, and oriented in alignment with the spatial signal, see the example in Fig. 2. In addition, the magnitude of the dominant spectral peak in relation to surrounding spectral peaks indicates the strength of the dominant signal. These features are utilized in the local analysis. A similar method based on local spectral analysis is described in [24], [25]. However, according to the evaluation in section III, there are distinct performance improvements in the proposed method.

The fundamental area size $L_{f}$ computed in Eq. 4 is used as a fundament in the local analysis, see Fig. 8. The size of the local area in the local analysis is $M \times M$, where $M$ is an odd-valued integer computed as

$$
M=2\left\lfloor\frac{k \cdot L_{f}}{2}+1\right\rfloor-1
$$

where the parameter $k$ is a design parameter that controls the number of fundamental periods enclosed by each local area.

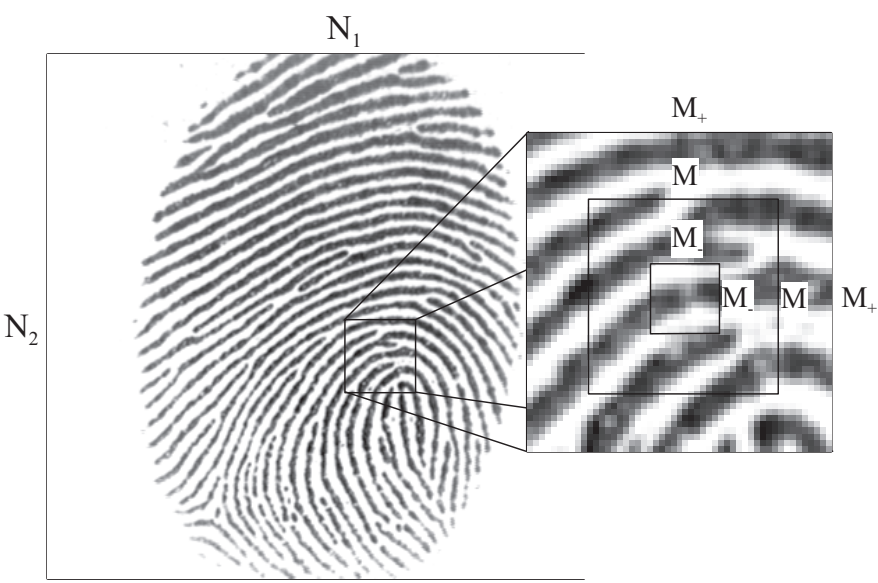

Fig. 9. Example of local area size $M \times M$, and corresponding sizes after growth $M_{+} \times M_{+}$and shrinkage $M_{-} \times M_{-}$.

Due to the local variability of a fingerprint, for example in regions around deltas, cores and minutiae where the fingerprint ridges are curved or when the local ridge frequency deviates from the estimated fundamental frequency $\omega_{f}$, two additional local area sizes are introduced. A larger local area size, denoted as $M_{+} \times M_{+}$, where $M_{+}=(1+\eta) \cdot M$, and a smaller local area size, denoted as $M_{-} \times M_{-}$, where $M_{-}=$ $(1-\eta) \cdot M$, are considered here. Note that both $M_{+}$and $M_{-}$ are forced to be odd-valued integers. The design parameter $\eta \in[0,1]$ defines the change, i.e., growth and shrinkage, of the larger and smaller area sizes in relation to the nominal local area size. It is stressed that all parameters used herein are functions of the automatically estimated fundamental area size $L_{f}$. Hence, the size of the local area, including the larger and smaller area sizes, automatically adapt to fingerprint and sensor variability. The approach to use three different sizes of the local area is illustrated in Fig. 9. Similar methods that incorporate multi-size windows or fingerprint image scaling are proposed in [26], [27], [28]. However, these methods adapt on a global scale, and this stands in contrast to the proposed method that adapts to each fingerprint on a local scale and thereby matches local variability better.

Each local area is centered around the point $\left(n_{1}, n_{2}\right)$ in the pre-processed image $X\left(n_{1}, n_{2}\right)$ according to

$$
J_{n_{1}, n_{2}}\left(m_{1}, m_{2}\right)=X\left(n_{1}+m_{1}, n_{2}+m_{2}\right),
$$

where $m_{1}=\left(-\frac{M-1}{2} \ldots \frac{M-1}{2}\right)$ and $m_{2}=\left(-\frac{M-1}{2} \ldots \frac{M-1}{2}\right)$ are coordinates in the local area. To clarify the presentation in the sequel, the notation of a local area, a local variable, or a local parameter is done without the local area sub-index $n_{1}, n_{2}$, i.e., $J_{n_{1}, n_{2}}\left(m_{1}, m_{2}\right) \equiv J\left(m_{1}, m_{2}\right)$.

The following steps are carried out for each local area in the local analysis:

1) A local dynamic range adjustment is proposed to be applied to each local area (see section II-C1).

2) A data-driven transformation is conducted in order to improve local spectral features estimation. The data for each local area is windowed and zero padded to the next larger power of two (see section II-C2). 


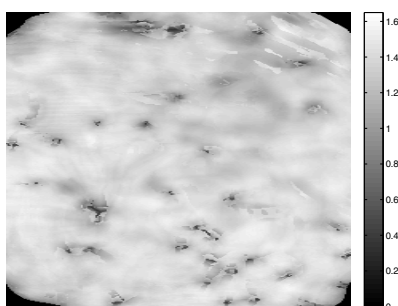

(a)

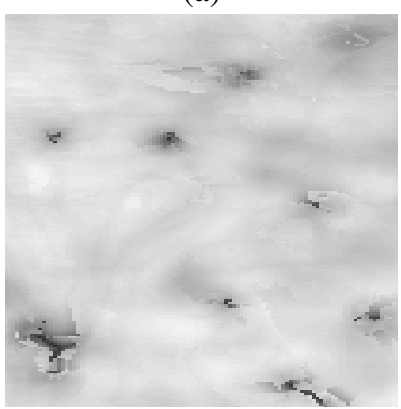

(c)

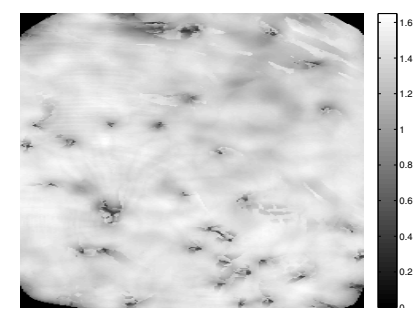

(b)

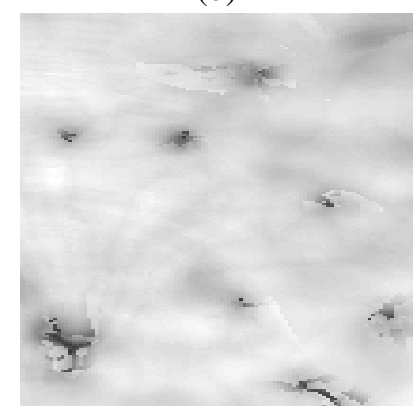

(d)
Fig. 10. The quality map $Q\left(n_{1}, n_{2}\right)$ obtained by using a local SMQT with $B=2$ (a) and $B=8$ (b). A close-up of quality map in (a) and (b) are shown in (c) and (d) respectively.

The two quality maps are close to identical for both cases.

3) A local magnitude spectrum is computed and the dominant spectral peak is located from which the local features frequency, orientation and magnitude are estimated.

4) A test if the local area needs to be reexamined, using a larger and a smaller size of the local area, is conducted. Steps 1-3 of the local analysis are repeated using these alternative area sizes if a reexamination is required.

1) Step 1 - Local dynamic range adjustment: Low quality fingerprint images usually consist of regions with a poor contrast between signal (i.e., fingerprint pattern), and background. This poor contrast may remain in some local areas even after global contrast enhancement. Local image areas having a poor contrast yield unsatisfactory local features extraction due to the low signal to noise ratio. A local contrast enhancement is therefore proposed herein by applying the SMQT dynamic range adjustment method on each local image area according to $H\left(m_{1}, m_{2}\right)=\mathrm{SMQT}_{2}\left\{J\left(m_{1}, m_{2}\right)\right\}$. It is noted that, the local analysis is based on local areas $J\left(m_{1}, m_{2}\right)$ of the globally SMQT-processed $X\left(n_{1}, n_{2}\right)$ image.

Through empirical analysis, it has been found that the SMQT used for local dynamic range adjustment only requires a two-bit representation, i.e., $B=2$, without degrading the local spectral features estimation. This stands out from the eight bits used in the dynamic range adjustment in the preprocessing stage (see section II-A). Quality maps, computed according to section II-C4, where local areas are enhanced by a two-bit SMQT $(B=2)$ and an eight-bit SMQT $(B=8)$ are illustrated in Fig. 10. The quality maps for the two dynamic ranges are close to identical, however, where the lower value $B=2$ requires less computational resources and is therefore preferred in a practical implementation.
2) Step 2 - Data transformation, windowing, zero padding: The local analysis uses a spatial window to suppress spectral side-lobes. The use of a window may yield feature estimation errors if a fingerprint valley is in the center of the local area since the window suppresses adjacent ridges. Hence, the dominant peak will be suppressed in the frequency spectrum as well. A simple test triggers a data-transformation that circumvents this problem. An arithmetic mean, denoted as $\bar{H}$, is estimated for data-values in and around the center of each local area according to

$$
\bar{H}=\frac{1}{(2 K+1)^{2}} \sum_{k_{1}=-K}^{K} \sum_{k_{2}=-K}^{K} H\left(k_{1}, k_{2}\right)
$$

where the parameter $K=\lfloor(M-1) / 4\rfloor$ controls the number of center points included in the estimate. The test and the following data transformation are conducted as follows

If $\bar{H}>2^{B-1}$ :

$$
H\left(m_{1}, m_{2}\right) \leftarrow 2^{B}-1-H\left(m_{1}, m_{2}\right),
$$

where $2^{B}-1$ denotes the maximal signal value for an image having $B$ bits of dynamic range, where $B=2$ due to the two-bit SMQT representation. The proposed test and transformation imply that, the sample values in the local area are inverted if the mean value is above half of the maximal dynamic range, which corresponds to having a fingerprint valley in the center of the local image area.

In order to improve local features extraction, the frequency spectrum has to have an adequate resolution. Therefore, each transformed local area is zero padded to the next higher power of two since an FFT is used to frequency-transform the image. To reduce the magnitude of spectral side-lobes, a twodimensional Hamming window is applied to the local area, smoothing the transition between data and the zero-padding.

It is noted that these steps are carried out for each local area, but where the local area indices $n_{1}$ and $n_{2}$ are omitted for clarity in the presentation.

3) Step 3 - Spectral features estimation: A local magnitude spectrum $G\left(\omega_{1}, \omega_{2}\right)=\left|\mathfrak{F}\left\{H\left(m_{1}, m_{2}\right)\right\}\right|$ is obtained by computing the modulus of the two-dimensional Fourier transform of the transformed, zero-padded and windowed local area $H\left(m_{1}, m_{2}\right)$. Spectral features include the magnitude $P_{D}$ and frequencies $\omega_{D, 1}, \omega_{D, 2}$ of the dominant spectral peak and the magnitude of the second largest spectral peak $P_{D_{2}}$.

A quality measure is computed based on the extracted features. The measure $\frac{P_{D}}{P_{\max }}$ quantifies the significance of the largest peak in relation to $P_{\max }$, the maximum magnitude possible including the bias of the window. The measure $\frac{P_{D_{2}}}{P_{D}}$ assesses the relationship between the two largest spectral peaks, $P_{D}$ and $P_{D_{2}}$, found in the magnitude spectrum of each local area. If the local area contains a dominant narrowband signal, such as a fingerprint pattern, $\frac{P_{D}}{P_{\max }}$ will be close to unity and $\frac{P_{D_{2}}}{P_{D}}$ will be close to zero. Hence, the measure

$$
Q=\frac{P_{D}}{P_{\max }}+\left(1-\frac{P_{D_{2}}}{P_{D}}\right)
$$


reflects the overall quality of the local area by combining these two measures. The measure $Q$ is thus referred to as a quality map where $Q=2$ implies best quality and $Q=0$ worst quality.

It is noted that each local area comprises a set of features, hence, the entire fingerprint image is represented, after the local analysis, by the feature maps $P_{D}\left(n_{1}, n_{2}\right), \omega_{D, 1}\left(n_{1}, n_{2}\right)$, $\omega_{D, 2}\left(n_{1}, n_{2}\right) P_{D_{2}}\left(n_{1}, n_{2}\right)$, and $Q\left(n_{1}, n_{2}\right)$.

4) Step 4 - Local area reexamination test: Some local areas need to be analyzed using a different local area size than the fundamental area size due to the variability in some regions of a fingerprint. Regions where the fingerprint ridges are curved, such as near cores, deltas and minutiae points, or where the local ridge frequency deviate from the estimated fundamental frequency $\omega_{f}$, may yield inaccurate spectral features estimates. These regions are reexamined using two additional sizes of the local area.

A reexamination of the local area is conducted if $Q \leq Q_{T}$, where $Q_{T}$ is a system design threshold. This means that steps 1-3 of the local adaptive analysis are repeated using the larger and smaller area sizes $M_{+}$and $M_{-}$, respectively. After the reexamination using the two new local area sizes is completed, similar quality scores $Q_{+}$and $Q_{-}$are calculated from the features from each respective stage. The final spectral features are chosen based on which of the measures $Q, Q_{+}$and $Q_{-}$ have the best quality (i.e., highest value).

\section{Matched Filtering}

A local area that contains a fingerprint image pattern is highly periodic and it therefore renders a strong dominant peak. The estimated local features $\omega_{D, 1}$ and $\omega_{D, 2}$ (from section II-C3) represent, respectively, the vertical and horizontal spatial frequencies of the local dominant spectral peak.

The estimated frequencies $\omega_{D, 1}$ and $\omega_{D, 2}$ are occasionally highly varying, e.g., where local curvature or irregularities such as cores, deltas and minutiae points in the fingerprint are located. A smoothing of these estimated frequencies is thus performed to reduce the impact of this noise. The smoothing is conducted on the polar coordinates $\omega_{D} \equiv \omega_{D}\left(n_{1}, n_{2}\right)$ and $\theta_{D} \equiv \theta_{D}\left(n_{1}, n_{2}\right)$ instead of the Cartesian coordinates $\omega_{D, 1}$ and $\omega_{D, 2}$, related as $\omega_{D}=\sqrt{\omega_{D, 1}^{2}+\omega_{D, 2}^{2}}$ and $\theta_{D}=$ $\arctan \left(\frac{\omega_{D, 2}}{\omega_{D, 1}}\right)$. The smoothing is performed by filtering $\omega_{D}$ and $\theta_{D}$ using order statistical filters, so called $\alpha$-trimmed mean filter [29], along the $n_{1}, n_{2}$-dimensions.

The $\alpha$-trimmed mean filter uses an observation window, of size $2 L+1 \times 2 L+1$ where $L=\left\lfloor\gamma \cdot L_{f}\right\rfloor$ and $\gamma$ is a design parameter. The sample values within the observation window are sorted and arranged into a column-vector containing $(2 L+$ $1)^{2}$ values. The output value of the filter is the mean of the $(1-$ $\alpha) \cdot(2 L+1)^{2}$ central sample values in the sorted vector, i.e., $\alpha$. $(2 L+1)^{2}$ extreme values at the beginning and the rear of the sorted vector are excluded from the mean. The parameter $\alpha$ is a design parameter. It is stressed that all parameters used herein are functions of the automatically estimated fundamental area size $L_{f}$. Hence, the size and shape of the order statistical filter, automatically adapt to fingerprint and sensor variability.
The polar angle map, $\theta_{D}$, is phase-wrapped around the values 0 and $\pi$ before smoothing by the order statistical filter to avoid irregular results, and the phase is reconstructed after the filtering. The smoothed polar coordinates are denoted as $\tilde{\omega}_{D}$ and $\tilde{\theta}_{D}$, respectively, and the corresponding smoothed Cartesian frequencies are thus $\tilde{\omega}_{D, 1}=\tilde{\omega}_{D} \cdot \cos \tilde{\theta}_{D}$ and $\tilde{\omega}_{D, 2}=\tilde{\omega}_{D} \cdot \sin \tilde{\theta}_{D}$.

The smoothed spatial frequencies are used to construct a filter $f\left(m_{1}, m_{2}\right)$, where $\left(m_{1}, m_{2}\right) \in[-N, N]$, matched to the local area at hand. The size of the filter is selected as $2 N+$ $1 \times 2 N+1$, where

$$
N=\operatorname{round}\left\{\frac{\pi}{\max \left(\left|\tilde{\omega}_{D, 1}\right|,\left|\tilde{\omega}_{D, 2}\right|\right)}\right\},
$$

i.e., rounded to the nearest integer value. The filter comprises a basis function $\phi\left(m_{1}, m_{2}\right)$ tapered with a spatially orthogonal tapering function $\tau\left(m_{1}, m_{2}\right)$, according to

$$
f\left(m_{1}, m_{2}\right)=\phi\left(m_{1}, m_{2}\right) \cdot \tau\left(m_{1}, m_{2}\right) .
$$

The basis function is a cosine function aligned to the frequency and orientation of the dominant peak, i.e.,

$$
\phi\left(m_{1}, m_{2}\right)=\cos \left(\tilde{\omega}_{D, 1} \cdot m_{1}+\tilde{\omega}_{D, 2} \cdot m_{2}\right),
$$

and the tapering function is orthogonal thereto

$$
\tau\left(m_{1}, m_{2}\right)=\cos \left(C \cdot\left(\tilde{\omega}_{D, 2} \cdot m_{1}-\tilde{\omega}_{D, 1} \cdot m_{2}\right)\right) .
$$

Following [2], [3], $C$ is a scalar variable used to stretch the tapering function so that it has the value 0.25 where the basis function (Eq. 12) intersects the filter boundary, $C$ is defined in [11]. The enhanced output image value at the point $\left(n_{1}, n_{2}\right)$ is the local area weighted by the corresponding matched filter

$$
Y\left(n_{1}, n_{2}\right)=\sum_{m_{1}=-N}^{N} \sum_{\substack{m_{2}=-N \\ X\left(n_{1}+m_{1}, n_{2}+m_{2}\right) .}}^{N} f_{n_{1}, n_{2}}\left(m_{1}, m_{2}\right) .
$$

\section{E. Image segmentation}

Fingerprint scanners have various sizes of the sensor area. This renders that fingerprint patterns obtained by a fingerprint scanner with a large sensor area only occupy a part of the image, as opposed to a scanner with a small sensor area. To suppress non-relevant parts of a fingerprint image, i.e., where there is no fingerprint data, a segmentation of the image is performed. In this paper, the segmentation of the fingerprint image is performed by applying a binary mask to the fingerprint image. The segmentation method used here is identical to that in [12], where the estimated spectral features from the local analysis are used to construct the binary mask. An additional post processing step is used to remove falsely segmented structured background from the binary mask, in accordance with [12]. The output image is the element-wise product of the binary mask and the matched filter output signal, i.e., $Y\left(n_{1}, n_{2}\right)$. 
TABLE I

VALUES OF THE DESIGN PARAMETERS CHOSEN IN THIS PAPER.

\begin{tabular}{cc}
\hline Parameter & Value \\
\hline$k$ & 3 \\
$\eta$ & 0.3 \\
$\alpha$ & 0.6 \\
$\gamma$ & 0.5 \\
$Q_{T}$ & 1 \\
\hline
\end{tabular}

\section{EVALUATION}

The proposed fingerprint enhancement algorithm is acting as a pre-processing stage to the NIST fingerprint recognition system consisting of a minutiae extractor (MINDTCT) and a minutiae matcher (BOZORTH3). The evaluation assesses the performance of the method, when the novel parts of each processing block are active respectively inactive, in section III-A. This part of the evaluation shows the improved performance due to each updated processing block in the proposed method. A complete performance evaluation where the proposed method is benchmarked towards the NIST implementation is provided in section III-B. The evaluation of the proposed method is carried out using the 12 databases included in FVC2000 [14], FVC2002 [15] and FVC2004 [16]. The evaluation results are quantified using Equal Error Rates (EER), in percentage, calculated according to [10]. The area above the Receiver Operating Curve (ROC), also known as the Area Above Curve (AAC) [30], [31], is used to complement the EER measure. In real applications, fingerprint matching systems often operate far from the EER point by decreasing the False Match Rate (FMR) in order to assure a high level of security. However, decreasing the FMR will cause the False Non Match Rate (FNMR) to increase, i.e., a larger amount of true matches are rejected. It is therefore of interest to evaluate the FNMR when FMR is $1 \%, 0.1 \%$ and $0 \%$, evaluated as FMR100, FMR1000, and ZeroFMR [32]. The performance measures EER, AAC, FMR100, FMR1000 and ZeroFMR, should be minimal and ideally zero. It is stressed that the proposed method is evaluated having the same parameter values for all databases, i.e., there is no parameter tuning to improve the performance for a specific database. In particular, the method parameter values used in the evaluation are listed in Table I. These are design parameters that are independent of specific fingerprints and sensors. The parameters are, except for $Q_{T}$, combined with the automatically estimated fundamental area size $L_{f}$ to adaptively steer the method. Hence, parameters such as the local area size or the order statistical filter size automatically adapt to fingerprint image variability. This adaptive behavior is identified as the main advantage of the present method. The design parameter $Q_{T}$ refers to a threshold of the quality map $Q$. Since $Q \in[0,2]$ for all fingerprints, the choice of $Q_{T}$ is also independent of specific fingerprints and sensors.

\section{A. Effects of processing blocks}

The purpose with this evaluation is to examine the effect of each of the four updated processing blocks of the new
TABLE II

PROCESSING BLOCKS 1 TO 4 TOGGLED ON AND OFF, THE CORRESPONDING PERFORMANCE METRICS AND COMPUTATIONAL IMPACT. BLOCK 1 REFERS TO THE SMQT 8 OF THE PRE-PROCESSING, BLOCK 2 REFERS TO THE MEDIAN FILTERING IN THE GLOBAL ANALYSIS, BLOCK 3 REFERS TO THE SMQT 2 OF THE LOCAL ANALYSIS, AND BLOCK 4 REFERS TO THE ORDER STATISTICAL FILTER IN THE MATCHED FILTERING BLOCK.

\begin{tabular}{|c|c|c|c|c|c|c|c|}
\hline & \multicolumn{4}{|c|}{ Processing blocks } & \multicolumn{2}{|c|}{$\begin{array}{c}\text { Performance } \\
\text { measure }\end{array}$} & $\begin{array}{c}\text { Computational } \\
\text { impact }\end{array}$ \\
\hline Variant & Block & lock & lock & lock 4 & EER & $\mathrm{AAC}$ & $\begin{array}{l}\text { Instructions } \\
\text { rel. to var. } 16\end{array}$ \\
\hline 1 & off & off & off & off & $4.9 \%$ & $2.4 \%$ & $65.15 \%$ \\
\hline 2 & off & off & off & on & $4.8 \%$ & $2.4 \%$ & $65.27 \%$ \\
\hline 3 & off & off & on & off & $2.7 \%$ & $0.3 \%$ & $99.82 \%$ \\
\hline 4 & off & off & on & on & $2.6 \%$ & $0.4 \%$ & $99.95 \%$ \\
\hline 5 & off & on & off & off & $4.8 \%$ & $2.4 \%$ & $65.15 \%$ \\
\hline 6 & off & on & off & on & $4.8 \%$ & $2.5 \%$ & $65.28 \%$ \\
\hline 7 & off & on & on & off & $2.8 \%$ & $0.3 \%$ & $99.83 \%$ \\
\hline 8 & off & on & on & on & $2.5 \%$ & $0.4 \%$ & $99.96 \%$ \\
\hline 9 & on & off & off & off & $2.6 \%$ & $0.4 \%$ & $65.19 \%$ \\
\hline 10 & on & off & off & on & $2.7 \%$ & $0.3 \%$ & $65.31 \%$ \\
\hline 11 & on & off & on & off & $2.6 \%$ & $0.2 \%$ & $99.87 \%$ \\
\hline 12 & on & off & on & on & $2.5 \%$ & $0.2 \%$ & $99.99 \%$ \\
\hline 13 & on & on & off & off & $2.6 \%$ & $0.4 \%$ & $65.20 \%$ \\
\hline 14 & on & on & off & on & $2.7 \%$ & $0.3 \%$ & $65.32 \%$ \\
\hline 15 & on & on & on & off & $2.6 \%$ & $0.2 \%$ & $99.87 \%$ \\
\hline 16 & on & on & on & on & $2.4 \%$ & $0.2 \%$ & $100.00 \%$ \\
\hline
\end{tabular}

proposed enhancement system, see Fig. 3. The evaluation is performed on the smaller B-set containing 10 users with eight samples per user in each of the 12 databases of the FVC2000, FVC2002 and FVC2004 databases. Each processing block is switched on and off yielding 16 different variants of the proposed method, where the mean EER and mean AAC over the 12 databases summarize the performance of each method variant. Results are presented in Table II, where block 1 refers to the $\mathrm{SMQT}_{8}$ of the pre-processing, block 2 refers to the median filtering in the global analysis, block 3 refers to the $\mathrm{SMQT}_{2}$ of the local analysis, and block 4 refers to the order statistical filter in the matched filtering block. The variant number 1 corresponds to the original unimproved method (see, [11], [12]) and variant number 16 corresponds to the proposed method, marked in bold face in Table II. The best EER and AAC performance is when all the novel processing blocks of the proposed method are active. In addition to the performance measures EER and AAC, the computational impact of each method variant is presented in relation to the proposed method, variant 16 . The computational impact is approximated using a reduced instruction set architecture. It is noted, that block 3, the local analysis $\mathrm{SMQT}_{2}$, adds most of the computational load compared to the other blocks. The blocks 1,2 and 4, introduce only a marginal additional processing.

A paired t-test is conducted for the 16 variants of the algorithm with the significance level $95 \%$ and where each algorithm variant was tested against variant 1 , the unimproved algorithm. The test revealed that block 1 and block 3 each, and combined, do contribute statistically significant to the reduction of the EER and AAC values compared to variant 1 , for all cases. This is also verified by considering the performance measures EER and AAC in Table II. A paired t-test between all combinations of variants revealed that block 2 and block 4 do bring a statistically significant reduction 

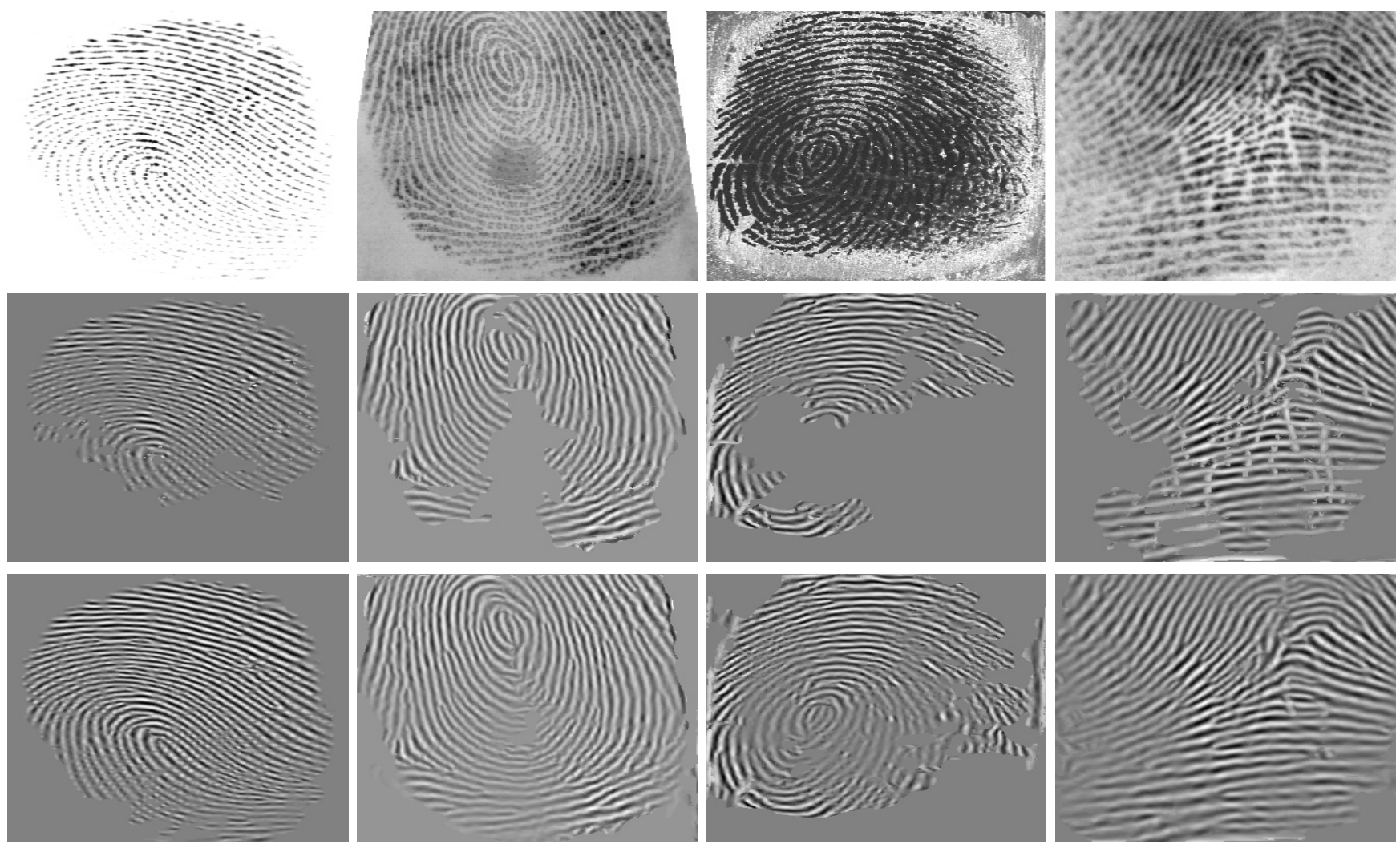

Fig. 11. Examples of the proposed fingerprint enhancement method. Top row: Original images, (from left) 46_2 FVC2004 db1a, 82_1 FVC2004 db2a, 50_2 FVC2000 db2a, and 69_1 FVC2000 db1a. Middle row: Enhancement with the original method (variant 1). Bottom row: Enhancement with the proposed method (variant 16).

in EER and AAC only if combined with either, or both, of block 1 and 3 . This is attributed to the fact that blocks 2 and 4 are intended to improve heavily degraded fingerprint images. For those images, the nonlinear blocks 2 and 4 provide a positive amplification of performance together with the nonlinear blocks 1 and 3 .

An illustration of the fingerprint enhancement with variant 1 and variant 16 of the proposed method is given in Fig. 11. The most pronounced visual effect is that fingerprints processed with the new method preserve larger parts of the original fingerprint, parts which were excluded in the original method. Another observation is that scars in the fingerprint are better removed (especially in the rightmost example), and this is attributed to a combination of the improved feature extraction and the added order statistical filtering of the matched filter design.

\section{B. Performance evaluation}

In order to be able to relate the results from this evaluation with results from earlier research, the FVC protocol of fingerprint verification, involving 2800 genuine matchings and 4950 imposter matchings, is adopted [10]. Results for the 12 databases with, and without, the proposed fingerprint enhancement method are provided in Table III. The proposed method improves the EER results for the NIST fingerprint recognition system on all databases. The improvement is specifically pronounced in the databases FVC2000, FVC2002 Db1a, Db3a and Db4a, and FVC2004 Db1a and Db2a, where the reduction in EER is between $12.5 \%$ and up to $50 \%$ in relation to the NIST method. The proposed method reduces the EER by as much as $50 \%$ in the database FVC2000 Db3a, which, according to [10], is the most difficult of the four FVC2000 databases.

In Table III, the AAC performance results show that the proposed method improves or provides the same AAC as the NIST method. The AAC is reduced in the databases FVC2000 Db1a, Db3a, Db4a, FVC2002 Db3a, Db4a, and FVC2004 Db1a, Db2a, Db4a by $24.3 \%$ to $66.7 \%$. Note that, even if AAC in FVC2002 Db1a, Db2a, and FVC2004 Db3a is unchanged by the proposed method, the relative improvement in EER, FMR100 and FMR1000 is increased, especially in FVC2004 Db3a. It is further observed that, for databases where the EER is improved also show improvements in the AAC.

The corresponding figures for FMR100 and FMR1000 follows the same improvement trend as for EER and AAC. The proposed method shows a significant performance improvement for all databases, where the FMR100 is reduced by up to $56.8 \%$ (in FVC2000 Db3a) and where FMR1000 is reduced by up to $49.2 \%$ (in FVC2000 Db3a). ZeroFMR is improved by $8.3 \%$ up to $48.0 \%$ in ten of the twelve databases. In two databases, FVC2000 Db1a and FVC2002 Db2a, the ZeroFMR is degraded by the proposed method in relation to NIST. To complement the data in Table III, so called Detection Error Tradeoff (DET) curves, which plots FMR against FNMR, for each database are illustrated in Fig. 12. The proposed method provides a lower FNMR for all FMR values for ten of the 
TABLE III

PERFORMANCE FOR THE NIST FINGERPRINT MATCHING METHOD (USING MINDTCT AND BOZORTH3) AND THE PROPOSED ENHANCEMENT METHOD FOR FVC DATABASES. RELATIVE IMPROVEMENT RELATES THE PROPOSED METHOD TOWARDS THE NIST METHOD. IMPROVED VALUES ARE MARKED IN BOLD FACE.

\begin{tabular}{|c|c|c|c|c|c|c|}
\hline & NIST & $\begin{array}{c}\text { NIST+ } \\
\text { proposed }\end{array}$ & $\begin{array}{c}\text { Relative } \\
\text { impr. }\end{array}$ & NIST & $\begin{array}{c}\text { NIST+ } \\
\text { propose }\end{array}$ & $\begin{array}{c}\text { Relative } \\
\text { impr. }\end{array}$ \\
\hline & \multicolumn{3}{|c|}{ FVC2000 Db1a } & \multicolumn{3}{|c|}{ FVC2000 Db2a } \\
\hline AAC & $3.2 \%$ & $1.1 \%$ & $65.6 \%$ & $1.5 \%$ & $1.4 \%$ & $6.7 \%$ \\
\hline EER & $5.8 \%$ & $3.5 \%$ & $39.7 \%$ & $4.4 \%$ & $3.5 \%$ & $20.5 \%$ \\
\hline FMR100 & $9.8 \%$ & $6.6 \%$ & $32.7 \%$ & $7.9 \%$ & $4.9 \%$ & $38.0 \%$ \\
\hline FMR 1000 & $13.5 \%$ & $12.0 \%$ & $13.0 \%$ & $12.1 \%$ & $8.0 \%$ & $33.9 \%$ \\
\hline \multirow[t]{2}{*}{ ZeroFMR } & $17.1 \%$ & $19.3 \%$ & $-11.4 \%$ & $20.2 \%$ & $10.5 \%$ & $48.0 \%$ \\
\hline & \multicolumn{3}{|c|}{ FVC2000 Db3a } & \multicolumn{3}{|c|}{ FVC2000 Db4a } \\
\hline$\overline{\mathrm{AAC}}$ & $3.3 \%$ & $1.1 \%$ & $66.7 \%$ & $1.6 \%$ & $0.9 \%$ & $43.8 \%$ \\
\hline EER & $7.6 \%$ & $3.8 \%$ & $50.0 \%$ & $5.5 \%$ & $3.7 \%$ & $32.7 \%$ \\
\hline FMR100 & $14.6 \%$ & $6.3 \%$ & $56.8 \%$ & $10.1 \%$ & $6.6 \%$ & $34.7 \%$ \\
\hline FMR1000 & $19.5 \%$ & $9.9 \%$ & $49.2 \%$ & $17.6 \%$ & $11.5 \%$ & $34.7 \%$ \\
\hline \multirow[t]{2}{*}{ ZeroFMR } & $24.9 \%$ & $16.8 \%$ & $32.5 \%$ & $25.5 \%$ & $14.4 \%$ & $43.5 \%$ \\
\hline & \multicolumn{3}{|c|}{ FVC2002 Db1a } & \multicolumn{3}{|c|}{ FVC2002 Db2a } \\
\hline$\overline{\mathrm{AAC}}$ & $1.3 \%$ & $1.3 \%$ & $0.0 \%$ & $1.0 \%$ & $1.0 \%$ & $\mathbf{0 . 0 \%}$ \\
\hline EER & $3.2 \%$ & $2.8 \%$ & $12.5 \%$ & $2.4 \%$ & $2.3 \%$ & $4.2 \%$ \\
\hline FMR100 & $5.0 \%$ & $4.0 \%$ & $20.0 \%$ & $3.5 \%$ & $3.2 \%$ & $8.6 \%$ \\
\hline FMR1000 & $7.2 \%$ & $6.1 \%$ & $15.3 \%$ & $5.3 \%$ & $4.8 \%$ & $9.4 \%$ \\
\hline \multirow[t]{2}{*}{ ZeroFMR } & $10.8 \%$ & $9.9 \%$ & $8.3 \%$ & $6.3 \%$ & $10.0 \%$ & $-37.0 \%$ \\
\hline & \multicolumn{3}{|c|}{ FVC2002 Db3a } & \multicolumn{3}{|c|}{ FVC2002 Db4a } \\
\hline AAC & $5.3 \%$ & $2.9 \%$ & $45.3 \%$ & $1.9 \%$ & $1.2 \%$ & $36.8 \%$ \\
\hline EER & $9.9 \%$ & $6.5 \%$ & $34.3 \%$ & $5.3 \%$ & $3.9 \%$ & $26.4 \%$ \\
\hline FMR100 & $18.4 \%$ & $11.7 \%$ & $36.4 \%$ & $8.8 \%$ & $6.8 \%$ & $22.7 \%$ \\
\hline FMR1000 & $28.6 \%$ & $18.6 \%$ & $35.0 \%$ & $12.7 \%$ & $10.0 \%$ & $21.3 \%$ \\
\hline \multirow[t]{2}{*}{ ZeroFMR } & $36.5 \%$ & $26.3 \%$ & $27.9 \%$ & $15.6 \%$ & $11.7 \%$ & $25.0 \%$ \\
\hline & \multicolumn{3}{|c|}{ FVC2004 Db1a } & \multicolumn{3}{|c|}{ FVC2004 Db2a } \\
\hline$\overline{\mathrm{AAC}}$ & $7.8 \%$ & $5.1 \%$ & $34.6 \%$ & $5.1 \%$ & $2.2 \%$ & $56.9 \%$ \\
\hline EER & $13.7 \%$ & $9.6 \%$ & $29.9 \%$ & $10.8 \%$ & $5.9 \%$ & $45.4 \%$ \\
\hline FMR100 & $26.8 \%$ & $18.9 \%$ & $29.5 \%$ & $19.9 \%$ & $10.5 \%$ & $47.2 \%$ \\
\hline FMR1000 & $35.3 \%$ & $26.4 \%$ & $25.2 \%$ & $26.6 \%$ & $17.5 \%$ & $34.2 \%$ \\
\hline \multirow[t]{2}{*}{ ZeroFMR } & $48.7 \%$ & $30.9 \%$ & $36.6 \%$ & $31.0 \%$ & $22.9 \%$ & $26.1 \%$ \\
\hline & \multicolumn{3}{|c|}{ FVC2004 Db3a } & \multicolumn{3}{|c|}{ FVC2004 Db4a } \\
\hline$\overline{\mathrm{AAC}}$ & $2.1 \%$ & $2.1 \%$ & $0.0 \%$ & $3.7 \%$ & $2.8 \%$ & $24.3 \%$ \\
\hline EER & $6.6 \%$ & $6.2 \%$ & $6.1 \%$ & $7.1 \%$ & $6.6 \%$ & $7.0 \%$ \\
\hline FMR100 & $15.1 \%$ & $12.8 \%$ & $15.2 \%$ & $12.3 \%$ & $10.9 \%$ & $11.4 \%$ \\
\hline FMR1000 & $29.7 \%$ & $19.6 \%$ & $34.0 \%$ & $19.1 \%$ & $14.5 \%$ & $24.1 \%$ \\
\hline ZeroFMR & $39.8 \%$ & $24.5 \%$ & $38.4 \%$ & $22.8 \%$ & $15.9 \%$ & $30.3 \%$ \\
\hline
\end{tabular}

twelve databases, i.e., the databases FVC2000 Db2a, Db3a, Db4a, FVC2002 Db1a, Db3a, Db4a, and FVC2004 Db1a, Db2a, Db3a, Db4a. The DET curve for database FVC2000 Db1a is improved for the proposed method when FMR exceeds $8 \cdot 10^{-4}$, and the DET curve is improved for database FVC2002 Db2a when FMR exceeds $4.5 \cdot 10^{-4}$.

The performance provided by the proposed method is put in relation to other published fingerprint image enhancement methods evaluated on FVC databases in a NIST-related manner. The method presented by Chikkerur et. al. [24] demonstrated $24.6 \%$ and $23.1 \%$ of relative improvement for EER and FMR100, respectively, on the FVC2002 Db3a database in comparison to the NIST method. This should be contrasted to the proposed method (see Table III) which reduces EER by $34.3 \%$ and FMR 100 by $36.4 \%$ on the same database. The fingerprint enhancement method proposed by Fronthaler et. al. [27], [28] showed an improvement of EER compared to the methods by Hong et. al. [4] and Chikkerur et. al. [25] for the databases in FVC2004. The relative EER improvement compared to the NIST method on FVC2004 Db1a to Db4a are $17.2 \%, 13.7 \%, 19.3 \%$ and $4.1 \%$, respectively. The corresponding relative EER improvement of the proposed method in relation to NIST is $29.9 \%, 45.4 \%, 6.1 \%$ and $7.0 \%$ for the same databases, i.e., our method shows an improvement for three of the four databases in FVC2004 in relation to [28]. A recently published method by Gottschlich [7] demonstrates an improvement of EER in comparison to [28] for the same databases. The different settings of curved Gabor filters in [7] exhibit relative EER improvement compared to the NIST method between $27.6 \%$ to $33.1 \%$ for Db1a, $33.6 \%$ to $37.9 \%$ for $\mathrm{Db} 2 \mathrm{a}, 17.7 \%$ to $22.6 \%$ for $\mathrm{Db} 3 \mathrm{a}$, and $11.0 \%$ to $17.8 \%$ for $\mathrm{Db} 4 \mathrm{a}$ respectively. The combination of curved Gabor filters and oriented diffusion filtering yields relative EER improvement compared to the NIST method between $35.9 \%$ to $38.6 \%$ for Db1a, $47.3 \%$ to $54.7 \%$ for Db2a, $32.3 \%$ to $45.2 \%$ for $\mathrm{Db} 3 \mathrm{a}$, and $26.0 \%$ to $32.9 \%$ for $\mathrm{Db} 4 \mathrm{a}$ respectively. The drawback of the method in [7] is its immense computational cost, estimated to be up to seven times more than the method proposed in this paper, which inhibits its practical usability in some applications. The estimation is based on the difference in number of instructions that are required to process one image. The number of instructions in our method is obtained by applying a reduced instruction set methodology. For the algorithm in [7], the specified hardware with processing time was used to approximate the number of instructions. In comparison to [7], the improvement of the proposed method for Db1a lies in between the performance of the curved Gabor filter method and NIST's method, and above the method in [7] for Db2a. In relation to the combined method in [7], the performance of the method proposed in this paper is close for Db2a.

\section{DISCUSSION}

The quality of fingerprint images and fingerprint sensor characteristics have a great influence on the performance of a fingerprint matching system. It is therefore common to employ fingerprint enhancement to increase the image quality and to improve the matching performance. In this paper, the proposed enhancement method is compared with three similar methods based on contextual filtering. Contextual filtering methods are dependent on locally estimated features such as fingerprint ridge frequency, orientation, and curvature, where these features are used to perform matched filtering locally. The feature estimation accuracy constrains how well these methods work, and one dominating factor is the size of the analysis window, i.e., the local area size, in relation to the characteristics of the fingerprint image.

The method proposed in this paper performs better than the method proposed by Chikkerur et. al. [24] on the FVC2002 Db3a database. Both methods are based on the same principle. That is, they estimate the local features by frequency analysis. The main difference is that the method in [24] has a block based processing with a fixed size of the local area and do not employ the adaptive window size that is used in our work, leading to unsatisfactory feature estimates. Also the method does not employ any nonlinear contrast enhancement on a global- or a local level.

The proposed method shows an improvement on three out of four FVC2004 databases in comparison to the method pro- 

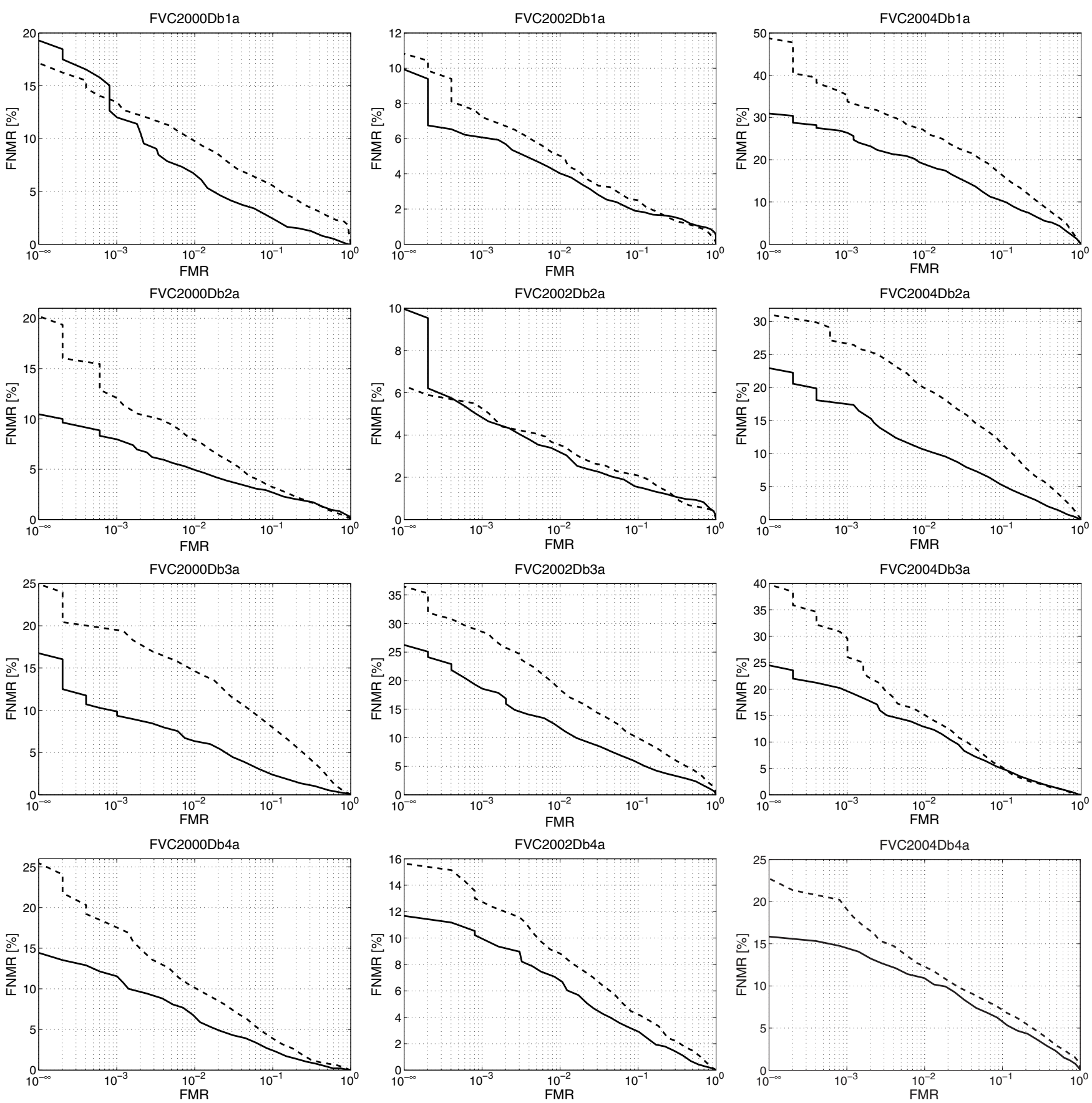

Fig. 12. DET curves for the NIST plus the proposed enhancement method (solid) and the NIST method (dashed) for FVC databases. Note that $10^{-\infty}$ denotes the ZeroFMR.

posed by Fronthaler et. al. [28]. The pyramid decomposition of the fingerprint image in [28] is to some extent related to the multi-size window reexamination in our paper. The main restriction of the pyramid decomposition approach is that it is based on the size of the image and not on the image content, i.e., the fingerprint characteristics. Enhancement of the fingerprints with a fundamental ridge frequency near or outside of the assumed boundary ridge frequency will be reduced. The application of the proposed estimated fundamental frequency in the pyramid decomposition could further improve the performance of the method in [28].

Another recent published method is proposed by
Gottschlich [7]. In this paper, curved Gabor filters are investigated. The curved Gabor method has an advantage compared to the linear methods explored here and in [24] and [28] due the possibility to follow curved patterns. However, this comes with an additional computational cost. The paper reports a performance improvement of the FVC2004 database compared to [24] and [28]. A possible performance improvement could involve the use of curved Gabor filters [7] in combination with the adaptive windows size and the nonlinear contrast enhancement proposed in this paper. 


\section{Summary And CONClusions}

This paper presents an adaptive fingerprint enhancement method. The method extends previous work by focusing on pre-processing of data on a global and a local level. A pre-processing using the non-linear SMQT dynamic range adjustment method is used to enhance the global contrast of the fingerprint image prior to further processing. Estimation of the fundamental frequency of the fingerprint image is improved in the global analysis by utilizing a median filter leading to a robust estimation of the local area size. A low-order SMQT dynamic range adjustment is conducted locally in order to achieve reliable features extraction used in the matched filter design and in the image segmentation. The matched filter block is improved by applying order statistical filtering to the extracted features, thus reducing spurious outliers in the feature data. The proposed method combines and updates existing processing blocks into a new and robust fingerprint enhancement system. The updated processing blocks lead to a drastically increased method performance where the EER is improved by a factor two, and the AAC is improved by a factor 12 , in relation to the original method (see variants 1 and 16 in Table II). The proposed method improves the performance in relation to the NIST method, and this is particularly pronounced on fingerprint images having a low image quality. The evaluation results indicate that the method is able to adapt to varying fingerprint image qualities, and it is stressed that the proposed method has not been tuned in favor towards any database. Rather, one global configuration has been used during the evaluation campaign. The proposed algorithm is insensitive to the varying characteristics of fingerprint images obtained by different sensors. A performance improvement is observed on databases with bad quality fingerprints (FVC2000 Db3a and FVC2004 Db2a). The ability of the proposed method to adapt to various images is emphasized in the results obtained on the FVC2000 Db3a database where the users' age ranges from 5 to 73 years.

A possible future research direction is to perform a detailed and systematic analysis of the impact of the different chosen design parameters (see, Table I). Furthermore, various optimizations of the implemented processing steps could reduce the number of instructions required by the proposed method.

\section{REFERENCES}

[1] D. Maltoni, D. Maio, A.K. Jain, and S. Prabhakar, Handbook of Fingerprint Recognition. Springer; 2nd edition, 2009, ISBN 978-184882-253-5.

[2] L. O'Gorman and J.V. Nickerson, "Matched filter design for fingerprint image enhancement," in Proc. Int. Conf. on Acoustic Speech and Signal Processing, 1988, pp. 916-919.

[3] L. O'Gorman and J. Nickerson, "An approach to fingerprint filter design,” Pattern Recognition, vol. 22, no. 1, pp. 29-38, 1989.

[4] L. Hong, Y. Wan, and A. Jain, "Fingerprint image enhancement: Algorithm and performance evaluation," IEEE Transactions on Pattern Analysis and Machine Intelligence, vol. 20, no. 8, pp. 777-790, 1998.

[5] B.G. Sherlock, D.M. Monro, and K. Millard, "Fingerprint enhancement by directional fourier filtering," Vision, Image and Signal Processing, IEE Proceedings-, vol. 141, no. 2, pp. 87-94, 1994.

[6] M. Tico, M. Vehvilainen, and J. Saarinen, "A method of fingerprint image enhancement based on second directional derivatives," in IEEE International Conference on Acoustics, Speech, and Signal Processing (ICASSP), 2005, pp. 985-988.
[7] C. Gottschlich, "Curved regions based ridge frequency estimation and curved gabor filters for fingerprint image enhancement," IEEE Transactions on Image Processing, vol. PP, no. 99, pp. 1-1, 2011.

[8] A. Willis and L. Myers, "A cost-effective fingerprint recognition system for use with low-quality prints and damaged fingertips," Pattern Recognition, vol. 34, no. 2, pp. 255-270, 2001.

[9] A. Uhl and P. Wild, "Comparing verification performance of kids and adults for fingerprint, palmprint, hand-geometry and digitprint biometrics," in IEEE 3rd International Conference on Biometrics: Theory Applications, and Systems, (BTAS), 2009, pp. 1-6.

[10] D. Maio, D. Maltoni, R. Cappelli, J. Wayman, and A. Jain, "Fvc2000: fingerprint verification competition," IEEE Transactions on Pattern Analysis and Machine Intelligence, vol. 24, no. 3, pp. 402-412, 2002.

[11] J. Ström Bartůněk, M. Nilsson, J. Nordberg, and I. Claesson, "Adaptive fingerprint binarization by frequency domain analysis," in IEEE Fortieth Asilomar Conference on Signals, Systems and Computers, 2006, pp. 598-602.

[12] — "Improved adaptive fingerprint binarization," in IEEE Congress on Image and Signal Processing, 2008, pp. 756-760.

[13] NIST home page for NBIS, "http://www.nist.gov/itl/iad/ig/nbis.cfm," 2010.

[14] FVC2000, "http://bias.csr.unibo.it/fvc2000/," 2010

[15] FVC2002, "http://bias.csr.unibo.it/fvc2002/," 2010.

[16] FVC2004, "http://bias.csr.unibo.it/fvc2004/," 2010

[17] M. Nilsson, M. Dahl, and I. Claesson, "The successive mean quantization transform," in IEEE International Conference on Acoustic, Speech, and Signal Processing (ICASSP), vol. 4, March 2005, pp. 429-432.

[18] _ "Gray-scale image enhancement using the smqt," in IEEE International Conference on Image Processing (ICIP), vol. 1, September 2005, pp. 933-936.

[19] R. Seshadri and Y. Avulapati, "Fingerprint image enhancement using successive mean quantization transform," International Journal of Computer Science and Information Security, vol. 8, no. 5, pp. 54-58, 2010.

[20] Y. Chen, S. Dass, and A. Jain, "Fingerprint quality indices for predicting authentication performance," in Proc. Int. Conf. on Audio- and VideoBased Biometric Person Authentication (5th), 2005, pp. 160-170.

[21] B. Lee, J. Moon, and H. Kim, "A novel measure of fingerprint image quality using the fourier spectrum," in Proc. SPIE Conf. on Biometric Technology for Human Identification II, vol. 5779, 2005, pp. 105-112.

[22] J. Libert, J. Grantham, and S. Orandi, A $1 D$ Spectral Image Validation/Verification Metric for Fingerprints. NISTIT 7599, 2009.

[23] J.S. Lim, Two-Dimensional Signal and Image Processing. PrenticeHall; 1st edition, 1990, ISBN 0-13-935322-4.

[24] S. Chikkerur, A.N. Cartwright, and V. Govindaraju, "Fingerprint enhancement using stft analysis," Pattern Recognition, vol. 40, no. 1, pp. 198-211, 2007.

[25] S. Chikkerur, V. Govindaraju, and A. N. Cartwright, "Fingerprint image enhancement using stft analysis," in International Conference on Advances in Pattern Recognition (ICAPR), vol. 2, 2005, pp. 20-29.

[26] C. Wu and V. Govindaraju, "Singularity preserving fingerprint image adaptive filtering," in IEEE International Conference on Image Processing, (ICIP), 2006, pp. 313-316.

[27] H. Fronthaler, K. Kollreider, and J. Bigun, "Pyramid-based image enhancement of fingerprints," in IEEE Workshop on Automatic Identification Advanced Technologies, 2007, pp. 45 - 50.

[28] —, "Local features for enhancement and minutiae extraction in fingerprints," IEEE Transactions on Image Processing, vol. 17, no. 3, pp. 354-363, 2008.

[29] I. Pitas and A.N. Venetsanopoulos, Nonlinear Digital Filters: Principles and Applications. Springer; 1st edition, 1990, ISBN 0-7923-9049-0.

[30] S. Gao, C.H. Lee, and J.H. Lim, "An ensemble classifier learning approach to roc optimization," in 18th International Conference on Pattern Recognition, (ICPR), vol. 2, 2006, pp. 679-682.

[31] K. Toh, J. Kim, and S. Lee, "Maximizing area under roc curve for biometric scores fusion," Pattern Recognition, vol. 41, no. 11, pp. 33733392, 2008.

[32] R. Cappelli, D. Maio, D. Maltoni, J. Wayman, and A. Jain, "Performance evaluation of fingerprint verification systems," IEEE Transactions on Pattern Analysis and Machine Intelligence, vol. 28, no. 1, pp. 3-18, 2006. 


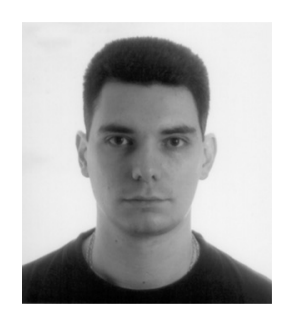

Josef Ström Bartůněk received the M.Sc.E.E. degree with specialization in signal processing from Blekinge Institute of Technology, Sweden, in 2005. $\mathrm{He}$ is currently pursuing $\mathrm{Ph} . \mathrm{D}$. degree in applied signal processing at School of Engineering, Blekinge Institute of Technology, Sweden. His research interests are related to signal processing, image processing, pattern recognition, and biometrics.

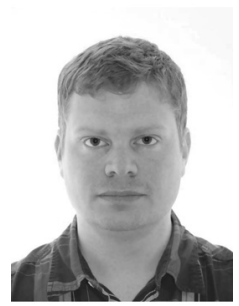

Mikael Nilsson (M'08) received the M.Sc.E.E and $\mathrm{Ph} . \mathrm{D}$. degree in applied Signal Processing from Blekinge Institute of Technology (BIT), in 2002 and 2007, respectively. He worked as an assistant professor at Blekinge Institute of Technology 20082011. Since November 2011 he is working as a Post. Doc. in Mathematics at the Centre for Mathematical Sciences in Lund University. His current research interests include pattern recognition, object detection in images, nonlinear signal processing and computer vision.

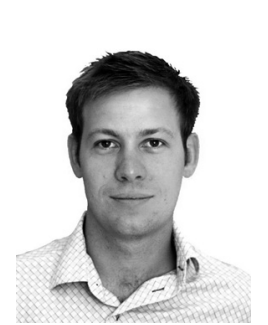

Benny Sällberg (M'04) was born in Sweden in 1979. He received his MSc degree and $\mathrm{PhD}$ in Telecommunications degrees at Blekinge institute of Technology, Sweden, in 2003 and 2008, respectively. He holds since 2009 a Post Doc position at the same university focusing on various signal processing applications of higher order methods. His research interests include statistical methods for signal enhancement in varios applications such as speech processing, image processing, and impulseradio ultra wide band. He has a pronounced focus on real-time signal processing in industrial applications.

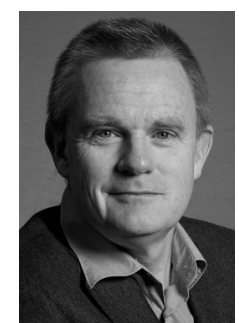

Ingvar Claesson (M'91) received the M.Sc.degree in 1980 and the Ph. D. degree in 1986 in Electrical Engineering at University of Lund, Sweden.

He was appointed Senior Lecturer in Telecommunication Theory at Lund University in 1986, and was appointed Associate Professor in 1992. In 1990, he was one of the founders of the Department of Signal Processing, Blekinge Institute of Technology, and is currently Head of Research and Principal Supervisor in Signal Processing. Since 1998, he holds the chair of Applied Signal Processing at Blekinge Institute of Technology, Ronneby, Sweden, and served as Research Dean 2005-2011.His current research interests are in adaptive signal processing, blind equalization, adaptive beamforming, speech enhancement, blind signal separation, active noise control, filter design, biometric signal and remote laboratories. 